\title{
Band Based Dynamic Link Adaptation for MC-CDMA Radio Interface
}

\author{
Asif Hamid, Reza Hoshyar, Rahim Tafazolli \\ Center for Communication Systems Research \\ University of Surrey \\ Guildford (GU2 7XH), UK \\ a.hamid@surrey.ac.uk
}

\begin{abstract}
This paper studies adaptive power allocation among sub-carriers in MC-CDMA. Due to intrinsic nature of MC-CDMA; Carrier Based power allocation schemes cause MAI (Multiple Access Interference) enhancements, hence fail at higher system loads. We propose a Band Based Dynamic Link Adaptation (BBDLA) scheme that preserves orthogonality (among users) by spreading user's signal only over a Band of adjacent $N$ sub-carriers $\left(N<N s c^{1}\right)$ lying within coherence bandwidth $\left(B_{c}\right)$ of the channel. Hence, it allows Band Based power allocation without causing any MAI. However, with only $N$ orthogonal users supported on a particular Band, BBDLA essentially proposes a hybrid of FDMA with MC-CDMA where Bands and transmit powers are optimally assigned to users by Base Station (in accordance with their channel state). Optimum Band allocation for BBDLA is found to be computationally intractable hence a sub-optimal heuristic approach is proposed with equal power distribution among all assigned Bands for each user. Effect of $B_{c}$ over choice of $N$ is studied and BBDLA with suitably chosen $N$, is shown to outperform other published Carrier Based power allocation schemes while it maintain almost single user BER performance up to $62 \%$ of full system loading.
\end{abstract}

Keywords; Multi-carrier CDMA, MC-CDMA; Transmit Power Allocation; Dynamic Carrier Allocation; Dynamic Carrier Selection; Link Adaptation; Bandwidth Allocation. Adaptive Frequency Hoping

\section{INTRODUCTION}

Recently, MC-CDMA (Multi-Carrier Code Division Multiple Access) has attracted significant interest as a multiple access method and has emerged as a potential candidate for $4 \mathrm{G}$ cellular systems [1]. This is mainly due to its capability of simultaneously exploiting the advantages of OFDM (Orthogonal Frequency Division Multiplexing) and CDMA (Code Division Multiple Access) to eliminate ISI (Inter Symbol Interference) caused by frequency selective fading, and to cope with narrowband interference. Thereby ensuring very high data rates required for $4 \mathrm{G}$ systems.

In the absence of CSI (Channel State Information) at transmitter, MC-CDMA relies on fixed FEC (Forward Error Correction) coding, bit interleaving [2] and frequency hopping [3] across the sub-carriers to exploit time and frequency diversity. However, if CSI is known at transmitter; the available frequency diversity can be exploited to its full extent

\footnotetext{
${ }^{1}$ Number of total available sub-carriers
}

by adapting the link level transmission parameters like subcarrier power, bit loading and sub-carrier frequency. This area of link adaptation has been exhaustively studied for simple multi-carrier systems like OFDM [4;5]. Though few realizably optimal solutions have also been proposed for MC-DS-CDMA [6;7] but MC-CDMA is largely without any comprehensive power allocation solution so far. In some of reported work [8;9] for MC-CDMA, authors have tried to exploit the Code Division Multiple Access nature of the system and (ignoring MAI issue) have suggested Carrier Based (i.e Chip Based) water filling type of power allocation schemes which are optimal for single user OFDM systems. In [10], although, authors have considered effect of MAI while formulating the problem, but then they have oversimplified problem by assuming that number of active users is much less than number of sub-carriers hence, have ignored MAI.

In MC-CDMA system multiple access is accomplished through spreading in frequency domain in an effort to absorb the frequency diversity to a greater extent. However, in multiuser scenario, its performance is greatly hampered by MAI caused by the frequency selectivity of the channel [11]. Therefore, Carrier Based power allocation schemes [8-10] derived by ignoring MAI, serves as an additional source of loss in multi-user orthogonality and deteriorates the BER performance to unacceptable levels. Hence all such schemes are applicable only to partially loaded systems. This is exactly due to this reason that optimal power solution derived (assuming MAI=0) by [10] is outperformed by a much simpler equal power allocation in higher SNR region where MAI interference dominates BER performance.

Keeping in view this underlying problem associated with power and sub-carrier allocation in MC-CDMA systems, we propose a novel Band Based Dynamic Link Adaptation scheme that not only adapts power along frequency but also preserve orthogonality among users by spreading the signal only over a band of adjacent sub-carriers $(\mathrm{N})$ lying within coherence bandwidth $\left(B_{c}\right)$ of the channel. Since, $N<N_{S C}$ (total available sub-carriers), therefore rest of the bandwidth is used to adaptively hop the signal along frequency in accordance with CSI. A complete framework for BBDLA is developed in this paper. Computational complexity associated with Optimum Band Allocation (OBA) for BBDLA is examined. Then Stochastically Fair Band Allocation (SFBA) is introduced as an heuristic approach to reduce the complexity and to ensure long term fairness among users. Effect of coherence bandwidth of the channel over the choice of $\mathrm{N}$ under different system loads is 
studied. BER performance of BBDLA with suitably chosen N, is compared against other published schemes for power and sub-carrier allocation in MC-CDMA. The remainder of this paper is organized as follows. In section II, a system model is described for conventional non-adaptive MC-CDMA which is modified in Section III as proposed by BBDLA. Simulation results are discussed in section IV. Paper concludes with a summary in section $\mathrm{V}$.

\section{SYSTEM MODEL}

Single cell MC-CDMA downlink is considered with simultaneously $\mathrm{J}$ active users. Perfect CSI from all users is assumed to be perfectly known to Base Station (BS) either, by an error free feedback channel (FDD mode) or, through reciprocity of channel (TDD mode). User's data rates are assumed equal and fixed with unit transmitter powers.

\section{A. Transmitter Architecture}

The generic MC-CDMA transmitter for $j^{\text {th }}$ user is shown in Figure 1. The input information stream $a_{j}$ (with bit duration $T_{b}$ ) is converted into $P$ parallel data sequences $\left[a_{j, 0}(i), a_{j, 1}(i), \ldots \ldots\right.$ $\left.a_{j, P}(i)\right]$. Thereby increasing symbol duration to $T_{s}=P T_{b}$. Subsequently, each of these sequences is spread over frequency domain using normalized Hadamard spreading codes $d_{j, m}$ with length $K_{M C}$. This spread data is used to modulate $N s c=P K_{M C}$ sub-carriers through Nsc point IDFT. Finally, cyclic prefix (CP) is used in guard interval $T_{g}$ after each OFDM symbol to overcome inter carrier interference. The complex equivalent low pass transmitted signal is given as

$$
\begin{gathered}
X_{j}(t)=\sum_{i=-\infty}^{+\infty} \sum_{p=0}^{P-1} \sum_{m=0}^{K} a_{j, p}(i) d_{j, m} \mu_{s}\left(t-i T_{s}^{\prime}\right) \\
\times e^{j^{\prime} 2 \pi\left(p K_{M C}+m\right) \Delta f^{\prime}\left(t-i T_{s}^{\prime}\right)} \\
\text { Where } \quad \sum_{m=0}^{K_{M C}-1}\left|d_{j, m}\right|^{2}=1
\end{gathered}
$$

$T_{S}^{\prime}$ is symbols duration at sub-carrier level, $\Delta f^{\prime}$ is the subcarrier spacing and $\mu_{s}$ is the rectangular symbol pulse waveform.

\section{B. Receiver Architecture}

Receiver architecture is shown in Figure 2. for the $j^{\text {th }}$ user. The received wave, after down conversion and $\mathrm{CP}$ removal is fed to Nsc point DFT for multi-carrier demodulation which calculates complex baseband Fourier coefficients $Y_{m, p}(i)$ at all Nsc subcarriers and time instant $t=i T_{s}{ }^{\prime}$. In downlink, the received signal at the $j^{\text {th }}$ receiver can be written as

$$
\begin{array}{r}
r_{j}(t)=\sum_{i=-\infty}^{+\infty} \sum_{p=0}^{P-1} \sum_{m=0}^{K_{M C^{-1}}(}\left(\sum_{j=1}^{J} a_{j, p}(i) d_{j, m} \mu_{S}\left(t-i T_{s}^{\prime}\right)\right) \times Z_{m, p, j(t)} \\
\times e^{j^{\prime} 2 \pi\left(p K_{M C}+m\right) \Delta f^{\prime}\left(t-i T_{s}^{\prime}\right)}+w(t)
\end{array}
$$

$$
D_{j, p}(i)=\sum_{m=0}^{K} d_{j, m}^{*} G_{j, p, m}(i) Y_{p, m}(i)
$$

Where

$$
Y_{p, m}(i)=\left[\sum_{j=0}^{J-1} a_{j, p} d_{j, m}\right] Z_{j, p, m}(i)+w_{p, m}(i)
$$

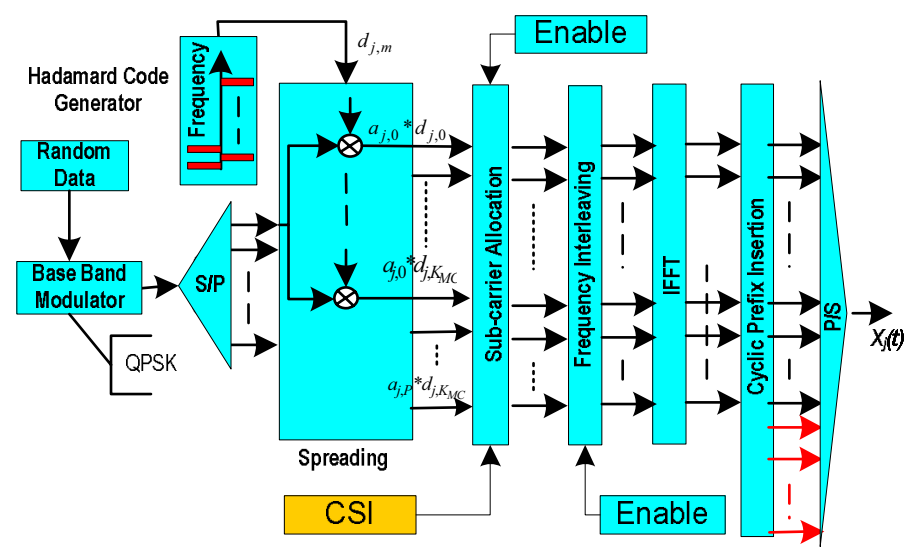

Figure 1. MC-CDMA Signal Generation

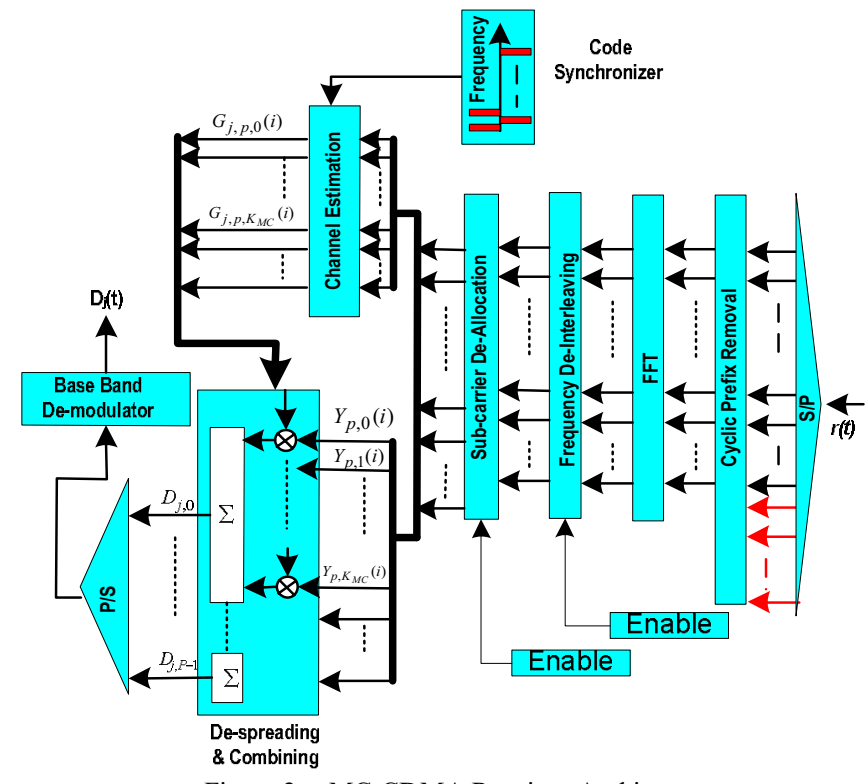

Figure 2. MC-CDMA Receiver Architectur

\section{BAND BASED DYNAMIC LINK ADAPTATION}

BBDLA scheme divides whole of the available transmission bandwidth $\Delta f^{\prime} \times N_{S C}$ into $\mathrm{K}$ smaller bands with $\mathrm{N}$ sub-carriers in each band such that $1 \leq N \leq K_{M C}$. Now, if $\mathrm{N}$ is chosen such that $N \times \Delta f^{\prime}<<B_{c}$, then $\mathrm{N}$ orthogonal users at the maximum may share each of these bands without undergoing any loss in their orthogonality due to frequency selective nature of the channel. Furthermore, since, $N<N_{S C}$, therefore rest of the bandwidth is used to adaptively hop the signal along frequency in accordance with CSI.

This new proposed scheme is actually a hybrid of " $\mathrm{K}$ " FDMA bands each one shared by $\mathrm{N}$ users in MC-CDMA fashion as shown in Figure 3. This reduction in spreading factor not only overcomes the MAI problem associated with multi-user MC-CDMA; but also provides an opportunity to jointly avail the benefits of the frequency diversity as well as multi-user diversity to its full extent by adaptively (with CSI)
Where $Z_{m, p, j}$ is the received complex envelop at the $\left(q=p K_{M C}+\right.$ $m)^{t h}$ sub-carrier of the $j^{\text {th }}$ user and $w$ is additive noise. Now, if $G_{j, p, m}(i)$ represnts the $j^{\text {th }}$ user total gain at the $q^{\text {th }}$ sub-carrier then decision variable for corresponding user can be written as 
allocating suitable bands to the users and adapting band powers. Now if $S_{i, k} \in\{1,0\}$ denotes the allocation and deallocation of the $K^{\text {th }}$ band to $j^{\text {th }}$ user respectively , then (1),(2),(4)and (5) needs to be modified as follows.

$$
\begin{aligned}
& X_{j}(t)=\sum_{i=-\infty}^{+\infty} \sum_{k=0}^{K-1} \sum_{m=0}^{N-1} b_{j, k}(i) d_{j, m} \\
& \quad \times \mu_{s}\left(t-i T_{s}^{\prime}\right) e^{j 2 \pi(k N+m) \Delta f^{\prime}\left(t-i T_{s}^{\prime}\right)} \\
& b_{j, k}= \begin{cases}a_{\mathrm{j}, \mathrm{p}} & \text { if } \mathrm{S}_{\mathrm{j}, \mathrm{k}}=1 \\
0 & \text { if } \mathrm{S}_{\mathrm{j}, \mathrm{k}}=0\end{cases} \\
& \text { Where } \\
& D_{j, k}\left(t=i T_{s}^{\prime}\right)=\sum_{m=0}^{N-1} d_{j, m}^{*} G_{j, k, m}(i) Y_{k, m}(i) \\
& Y_{k, m}(i)=\left[\begin{array}{l}
n_{k}-1 \\
\sum_{j=0} b_{j, k} d_{j, m}
\end{array}\right] Z_{j, k, m}(i)+w_{k, m}(i)
\end{aligned}
$$

Whereas, the $J \times K$ matrix $\mathbf{S}=\left[S_{j, k}\right]$ is the subcarrier allocation coefficients matrix, which is updated periodically with period of $T_{h}$ at transmitter such that:

$$
\begin{aligned}
\mathrm{n}_{\mathrm{k}} & =\sum_{\mathrm{j}=0}^{\mathrm{J}-1} \mathrm{~S}_{\mathrm{j}, \mathrm{k}} \leq N \quad \forall \mathrm{k} \\
\mathrm{S}_{\mathrm{j}} & =\sum_{\mathrm{k}=0}^{\mathrm{K}-1} \mathrm{~S}_{\mathrm{j}, \mathrm{k}}=P \quad \forall \mathrm{j}
\end{aligned}
$$

Where, $n_{k}, S_{j}$ are the total users allocated to $k^{\text {th }}$ band and total information symbols from $j^{\text {th }}$ user in one OFDM symbol respectively. It must be noted that total transmission power (assumed unity in our model) is equally divided among selected Bands. Although, this power could have been adapted in an optimal fashion however, we have made no such attempt following the findings of [13], which suggests that with suitable allocation of Bands, even Equal Power Allocation performs extremely close to Water-filling solution.

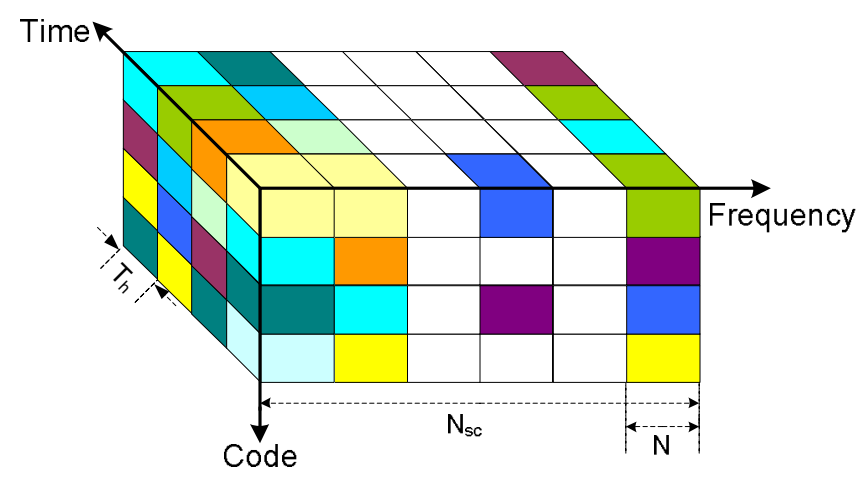

Figure 3. Adaptive MC-CDMA using BBDLA with CSI updation time period of $\mathrm{T}_{\mathrm{h}}$. Colours represent users with orthogonal codes. Total number of sub-carriers $=$ Nsc, Sub-carrier per MC-CDMA band $=\mathrm{N}$ and $\mathrm{K}=\mathrm{Nsc} / \mathrm{N}$

\section{A. Optimum Band Allocation}

Since, $\mathrm{P}$ bands are required for each user in order to meet its data rate requirements and only $\mathrm{N}$ out of $\mathrm{J}$ active user can be accommodated on each of the $\mathrm{K}$ bands. Therefore, optimum band allocation (where each user is allocated its favorite subcarriers) becomes a version of well known set portioning problem. Let $A=\left\{A_{1}, A_{2}, \ldots . . . A_{J}\right\}$ be any partition of the set of all available bands $B=\{1,2, \ldots \ldots . . K\}$ into $J$ subsets such that cardinality of $A_{j}=P$ and no $\mathrm{k} \in \mathrm{B}$ belongs to more than $N$ number of sub-sets of $A$. Since, the sub-sets of $A$ are not disjoint, therefore, this problem is very complex and computationally extensive to solve. It has been shown in [14] that size of solution space for such a problem with $\mathrm{J}$ users, $N_{s c}=128$ and $N=16$ is

$$
\mathrm{J} \times\left(\begin{array}{l}
\mathrm{N}_{\mathrm{sc}} \\
N
\end{array}\right)>10^{20}
$$

Therefore, a heuristic approach is taken to solve this problem and Stochastically Fair Band Allocation (SFBA) scheme is proposed that guarantees long term fairness among users.

\section{B. Stochastically Fair Band Allocation}

SFBA, at every updation period randomly selects users and let them choose their favorite bands among the available bands subject to (11) and (12). Complete algorithm is given below:

i. Initialize $\mathrm{S}_{\mathrm{j}, \mathrm{k}}=0, n_{k}=0 ; \forall \mathrm{j}, \mathrm{k}$

ii. Define power threshold $\mathrm{P}_{\text {th }}=[0,1]$

iii. Define set of active users $\mathrm{Q}=\{0,1 \ldots \ldots . \mathrm{J}-1\}$

iv. Randomly select a $\mathrm{j} \in \mathrm{Q}$ and define $\mathrm{C}_{\mathrm{th}}=\mathrm{N}$

v. Find $k \in \mathrm{X}=\{0,1 \ldots \ldots \ldots \mathrm{K}-1\}$ such that

- $\left|Z_{j, k, m}\right|^{2} \geq P_{t h}$ for $\mathrm{m}=1$ to $\mathrm{C}_{\mathrm{th}}$

- $\quad$ and $n_{k} \leq N$ and mark it as $k^{*}$

vi. If more than one $k^{*}$ are found, picks one with highest band gain i.e.

$$
k^{*} \leftarrow \arg \max _{k^{*}} \sum_{m=0}^{N-1}\left|Z_{j, k^{*}, m}\right|^{2}
$$

vii. Increment $n_{k}$ by 1 and delete $k^{*}$ from $\mathrm{X}$

viii. If no such $k^{*}$ is found, reduce $\mathrm{C}_{\mathrm{th}}$ by 1 and repeat steps v-vii until one such $k^{*}$ is found.

ix. Calculate $S_{j}$ using (12). If $S_{j}<P$, keep on repeating steps $\mathrm{v}$-ix until $\mathrm{S}_{\mathrm{j}}=\mathrm{P}$.

$\mathrm{X}$. Delete the $\mathrm{j}$ from $\mathrm{Q}$

xi. If $\mathrm{Q} \neq\{\}$ then repeat steps iv-xi all over again

\section{SIMUlations AND RESUlts}

Simulation parameters are shown below in Table I which have been adopted from MATRICE ${ }^{2}$ (MC-CDMA Transmission Techniques for Integrated Broadband Cellular Systems) specifications. First of all, in order to study the effect of coherence bandwidth of channel on the choice of $\mathrm{N}$ we have studied the BER performance in presence of BBDLA as function of band size $\mathrm{N}$ for different channel types (TABLE II. ). As shown in Figure 4. a sharp decline in system performance can be observed when value of $\mathrm{N}$ is increased beyond $N_{t h}$ which

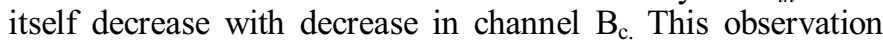
reinforces our hypothesis: "reduction in spreading factor such

${ }^{2}$ European-IST project could be accessed at http://www.istmatrice.org/ 
that $N \times \Delta f^{\prime}<<B_{c}$ preserve orthogonality among users hence improves performance". Interestingly the optimum (which minimizes BER in given scenario) value of $\mathrm{N}$ is found to be 1 for all channel realization. This observation promotes OFDMA as better candidate rather than MC-CDMA as along as CSI is known at transmitter. However, spread spectrum nature of MCCDMA will be advantageous to suppress Other Cell Interference (in multi-cell scenario) and narrow band interference especially in highly loaded system where freedom to maneuver around such jammed sub-carriers will be less. Therefore, we can safely opt for a MC-CDMA with reduced spreading factor $\mathrm{N}_{\text {th }}$ which gives a BER performance very close to OFDMA and don't completely strip off the system robustness against any possible interference.

TABLE I. SIMULATION PARAMETERS

\begin{tabular}{|c|l|c|c|}
\hline No & \multicolumn{1}{|c|}{ Parameter } & Symbol & Value \\
\hline 1. & RF carrier frequency & $\mathrm{F}_{\mathrm{c}}$ & $5 \mathrm{GHz}$ \\
\hline 2. & Baseband modulation & & QPSK \\
\hline 3. & No. of parallel branches & $\mathrm{P}$ & 23 \\
\hline 4. & Spreading codes & \multicolumn{2}{|c|}{ Hadamard Walsh } \\
\hline 5. & Spreading factor & $\mathrm{K}_{\mathrm{MC}}$ & 32 \\
\hline 6. & Total used sub-carriers & $\mathrm{N}_{\mathrm{sc}}$ & 736 \\
\hline 7. & FFT size & $\mathrm{N}_{\mathrm{FFT}}$ & 1024 \\
\hline 8. & Useful symbol duration & $\mathrm{T}_{\mathrm{s}}$ & $17.75 \mu \mathrm{s}$ \\
\hline 9. & Guard interval & $T_{g}$ & $3.75 \mu \mathrm{s}$ \\
\hline 10. & Symbol total duration & $T_{s}$ & $21.506 \mu \mathrm{s}$ \\
\hline 11. & Sampling frequency & $f_{s}$ & $57.6 \mathrm{MHz}$ \\
\hline 12. & Combining technique & \multicolumn{2}{|c|}{ MRC } \\
\hline 13. & CSI updation period & $T_{h}$ & $20 \mathrm{OFDM}$ \\
\hline
\end{tabular}

TABLE II. ChanNEl Delay PROFILES

\begin{tabular}{|c|c|c|c|}
\hline \multirow{2}{*}{ Channel } & \multicolumn{2}{|c|}{ Power Delay Profile } & $\begin{array}{c}\text { Bc } \\
{[\mathbf{M H z}]}\end{array}$ \\
\hline \multirow{2}{*}{$\mathrm{Ch} 1$} & $\mathrm{dp}$ & {$[0,110,190,410] \mathrm{ns}$} & \multirow{2}{*}{3.46} \\
\cline { 2 - 3 } & $\mathrm{pp}$ & {$[0,-9.7,-19.2,-22.8] \mathrm{dB}$} & \\
\hline \multirow{2}{*}{$\mathrm{Ch} 2$} & $\mathrm{dp}$ & {$[0,400,700,1000] \mathrm{ns}$} & \multirow{2}{*}{1.06} \\
\cline { 2 - 3 } & $\mathrm{pp}$ & {$[0,-9.7,-19.2,-22.8] \mathrm{dB}$} & \\
\hline \multirow{3}{*}{ BranE } & $\mathrm{dp}$ & {$[0,138.9,312.5,711.8,1284.7,1753.5] \mathrm{ns}$} & \multirow{2}{*}{0.81} \\
\cline { 2 - 3 } & $\mathrm{pp}$ & {$[-4.90,-0.28,0.10,-5.42,-13.45,-20.92] \mathrm{dB}$} & \\
\hline
\end{tabular}

dp: Channel delay profile, pp: Channel power profile, BranE is ETSI channel model for outdoor propagation at $5 \mathrm{GHz}$

Having obtained a suitable value of $\mathrm{N}=4$ for $\mathrm{BranE}$ channel, in Figure 5. we have compared BBDLA performance against a typical Carrier Based Dynamic Power and Carrier allocation (CBDPCA) proposed by many like [8-10] and simple bit interleaving [2] scheme. BER as function of system load is chosen as performance metric. It can be seen that interleaving improves system performance as compared to a conventional MC-CDMA system but only at lower system loads where diversity gain exploited by interleaving is more as compared to the additional MAI caused. However reverse is true for higher system loads. Similarly, CBDPCS outperforms the non-adaptive MC-CDMA at low system loads and its performance deteriorates sharply with system load which can be attributed to MAI enhancement by carrier based power allocation. However, in case of BBDLA the system load has almost negligible effect on performance until an appreciable system loading of $62 \%$ (users 20) beyond which BBDLA performance decreases slowly due to decreased available frequency adaptivity. However, even at full system load the BER performance stays at a reasonable value of $4 \times 10^{-4}$ at $\mathrm{Eb} / \mathrm{No}=12 \mathrm{~dB}$ for maximum Doppler shift of $\mathrm{Fd}=33.36 \mathrm{~Hz}$ that corresponds to a typical mobile user at speed of $7.2 \mathrm{Km} / \mathrm{h}$.

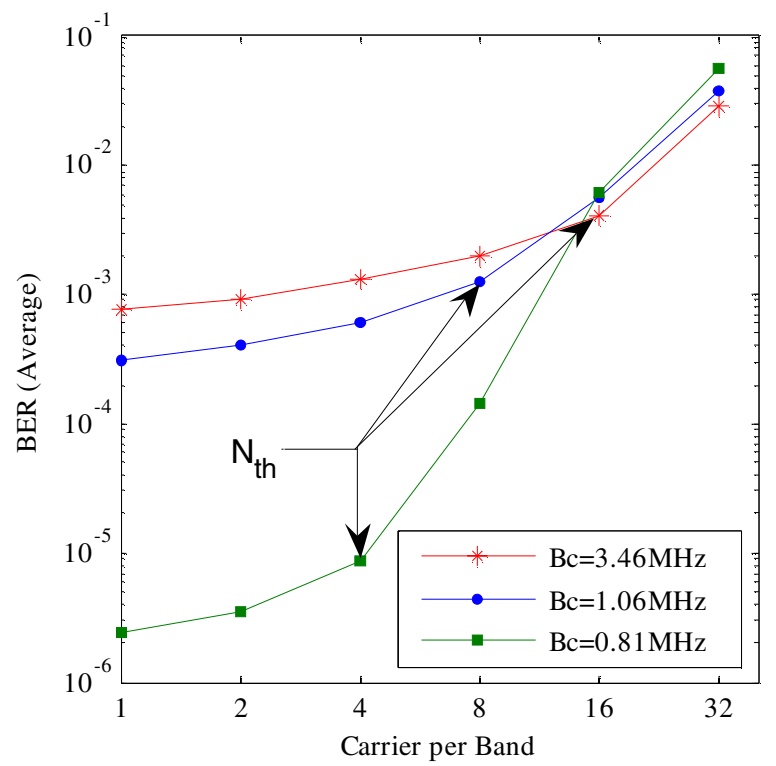

Figure 4. Effect of change in number of sub-carriers per band on the performance of BBDLA in different radio channels. Users $=5, \mathrm{Fd}=33.36 \mathrm{~Hz}$ ( a typical mobile user with speed of $7.2 \mathrm{~km} / \mathrm{h}$ ), Eb/No $=12 \mathrm{~dB}$

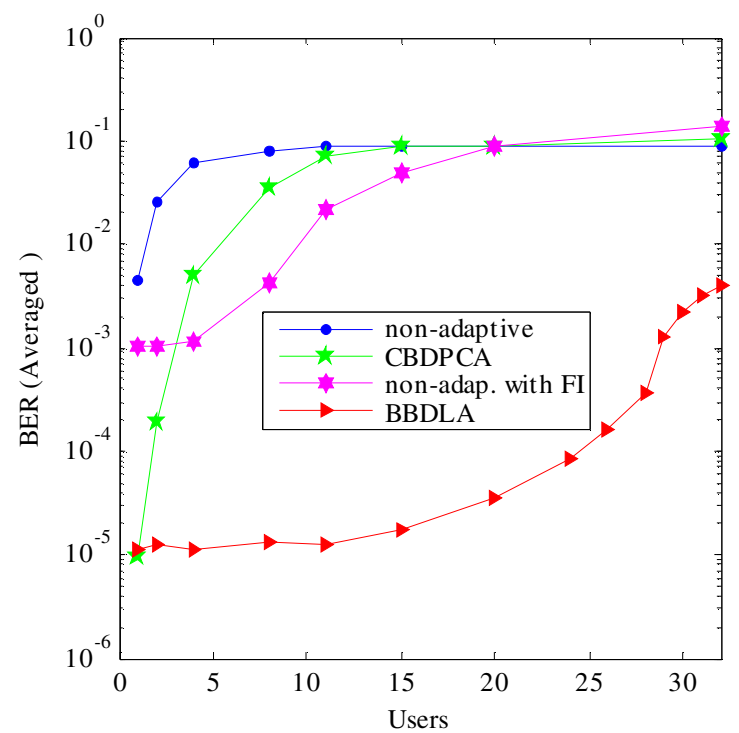

Figure 5. Performance comparison of BBDLA against Carrier Based Dynamic Carrier Allocation and non-adaptive MC-CDMA. BranE Channel, $\mathrm{Eb} / \mathrm{No}=12 \mathrm{~dB}, \mathrm{~N}=4$ (BBDLA), $\mathrm{Fd}=33.36 \mathrm{~Hz}$

In Figure 6. BER performance is studied as function of SNR and a comparison is drawn between BBDLA and nonadaptive MC-CDMA using frequency interleaving. It can be seen that performance of BBDLA (with system loading of $50 \%$ ) is remarkably higher than a single user non-adaptive MC-CDMA. Another rather interesting behavior of BBDLA is 
observed that at lower $\mathrm{Eb} / \mathrm{No}$ values, where it has out performed the non-adaptive MC-CDMA performance even in non fading AWGN channel which can be attributed to the SNR gains offered by the adaptive selection of bands.

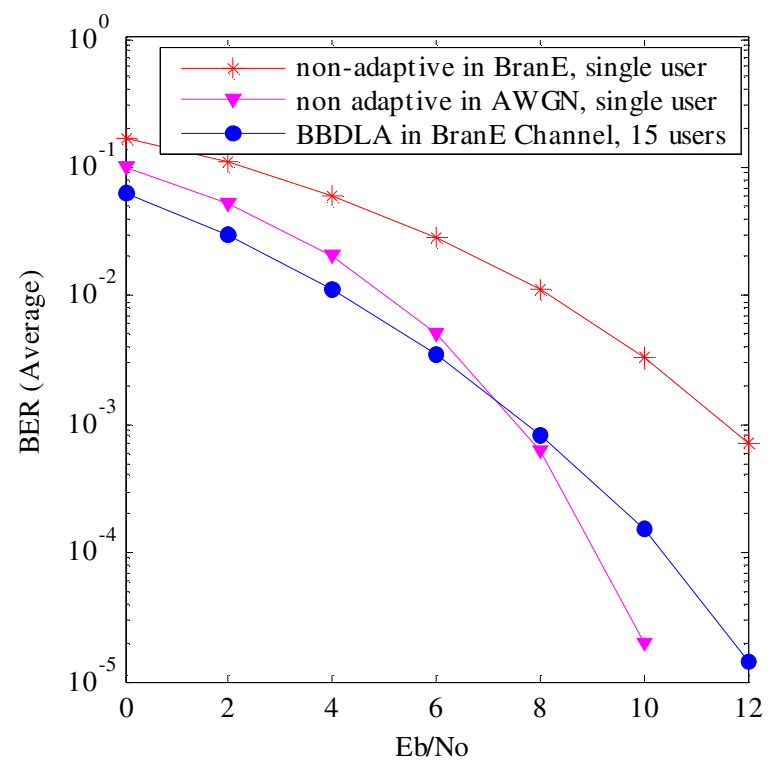

Figure 6. Performance comparison of BBDLA with $\mathrm{J}=15, \mathrm{~N}=4$ against single user non-adaptive MC-CDMA (using frequency interleaving) in BranE Channel as well as in AWGN Channel . Fd $=33.36 \mathrm{~Hz}$

\section{CONCLUSION}

We have shown that Carrier Based dynamic carrier and power allocation techniques, which substantially improve the performance in MAI free systems (like OFDMA) or where MAI is not caused by frequency selectivity of channel (like MC-DS-CDMA), can't be generalized to MC-CDMA (where MAI is strongly related to frequency selectivity of channel). Therefore, we have proposed BBDLA scheme for MC-CDMA, which not only allocate suitable bands to different users but also preserves orthogonality among users and hence keeps MAI to its statistical minimum. Although, with transmit power equally distributed among selected bands, BBDLA is shown to outperform the existing carrier based dynamic carrier allocation schemes derived ignoring the MAI enhancements caused by power allocation. This scheme approximately maintains a single user performance even at a system load of $62 \%$ beyond which its BER performance undergoes graceful degradation but even then stays at a reasonably acceptable value of $4 \times 10^{-4}$ at $\mathrm{Eb} / \mathrm{No}=12 \mathrm{~dB}$ in a typical outdoor mobile channel for a typical mobile user.

\section{REFERENCES}

[1] R. Prasad and S. Hara, "An overview of multi-carrier CDMA," ISSSTA 1996 - IEEE International Symposium on Spread Spectrum Techniques and Applications, vol. 1, 1996, pp. 107-114

[2] P. Xiaoming, A. S. Madhukumar, and F. P. S. Chin, "Performance studies of interleaving schemes for MC-CDMA systems," WCNC 2004 - IEEE Wireless Communications and Networking Conference, vol. 4, 2004, pp. 2081-2086

[3] M. Elkashlan and C. Leung, "Performance of frequency-hopping multicarrier CDMA on an uplink with correlated Rayleigh fading," GLOBECOM 2003 - IEEE Global Telecommunications Conference, vol. 6, 2003, pp. 3407-3411

[4] Y. W. Cheong, R. S. Cheng, K. B. Lataief, and R. D. Murch, "Multiuser OFDM with adaptive subcarrier, bit, and power allocation,"
IEEE Journal on Selected Areas in Communications, vol. 17, no. 10, pp. 1747-1758, 1999

[5] D. Kivanc, L. Guoqing, and L. Hui, "Computationally efficient bandwidth allocation and power control for OFDMA," IEEE Transactions on Wireless Communication, vol. 2, no. 6, pp. 1150$1158,2003$.

[6] H. K. Yun, S. Iickho, Y. Seokho, and R. P. So, "A multicarrier CDMA system with adaptive subchannel allocation for forward links," IEEE Transactions on Vehicular Technology, vol. 48, no. 5, pp. 1428-1436, 1999.

[7] C. Qingxin, E. S. Sousa, and S. Pasupathy, "Multicarrier CDMA with adaptive frequency hopping for mobile radio systems," IEEE Journal on Selected Areas in Communications, vol. 14, no. 9, pp. 1852-1858, 1996.

[8] Z. Yu and E. Gunawan, "Performance of MC-CDMA system using controlled MRC with power control in Rayleigh fading channel," IEE Electronics Letters, vol. 36, no. 8, pp. 752-753, 2000.

[9] Y. H. Lee and Y. Bar-Ness, "Transmission power adaptations in MCCDMA communications over Rayleigh fading channels," WCNC 2004 - IEEE Wireless Communications and Networking Conference, vol. 3, no. 1,2004 , pp. $1589-1594$

[10] Z. Jianming and Y. Bar-Ness, "Power allocation algorithm in MCCDMA," ICC 2002 - IEEE International Conference on Communication, vol. 2, 2002, pp. 931-935

[11] K. Jayong and H. Youngnam, "The effect of spreading factor in MCCDMA systems under multi-cell environments in correlated fading channels," vol. 7, 2004, pp. 4983-4987

[12] "'"QoS Concept and Architecture," 3rd Generation Partnership Project (3GPP), Tech. Spec. 23.107, V.4.0.0, 2000.,"2000.

[13] J. Jiho and B. L. Kwang, "Transmit power adaptation for multiuser OFDM systems," IEEE Journal on Selected Areas in Communications, vol. 21, no. 2, pp. 171-178, 2003.

[14] L. Chuxiang and W. Xiaodong, "Adaptive subchannel allocation in multiuser MC-CDMA systems," GLOBECOM 2004 - IEEE Global Telecommunications Conference, vol. 4, 2004, pp. 2503-2507 